OPEN ACCESS

Edited by:

Nunzio Itaco,

University of Campania Luigi Vanvitelli,

Reviewed by

Maria Colonna,

Laboratori Nazionali del Sud (INFN),

Kevin Fr

Michigan State University,

United States

*Correspondence:

Maria Piarulli

mpiarulli@physics.wustl.edu

Specialty section:

This article was submitted to Nuclear Physics,

a section of the journal

Frontiers in Physics

Received: 08 June 2020

Accepted: 28 June 2020

Published: 02 November 2020

Citation:

King GB, Andreoli L, Pastore $S$ and

Piarulli M (2020) Weak Transitions in

Light Nuclei. Front. Phys. 8:363.

doi: 10.3389/fphy.2020.00363

\section{Weak Transitions in Light Nuclei}

\author{
Garrett B. King ${ }^{1}$, Lorenzo Andreoli ${ }^{1}$, Saori Pastore ${ }^{1,2}$ and Maria Piarulli ${ }^{\text {1* }}$ \\ ${ }^{1}$ Department of Physics, Washington University, St. Louis, MO, United States, ${ }^{2}$ McDonnell Center for the Space Sciences at \\ Washington University, St. Louis, MO, United States
}

Nuclei are used for high-precision tests of the Standard Model and for studies of physics beyond the Standard Model. Without a thorough understanding of nuclei, we will not be able to meaningfully interpret the growing body of experimental data nor will we be able to disentangle new physics signals from underlying nuclear effects. This calls for accurate calculations of nuclear structure and reactions. In this work, we focus on electroweak decays in nuclei with mass number $A \leq 10$ and report on ab initio Quantum Monte Carlo calculations of reduced matrix elements entering beta decays and electron captures in nuclei with mass number $A \leq 10$. The many-body wave functions are calculated using selected Norfolk two- and three-nucleon potential models and associated one- and two-body axial currents at tree-level obtained from a chiral effective field theory with pions, nucleons, and $\Delta$. The agreement with the experimental data is satisfactory except for transitions in $A=8$ nuclei. In this specific case, the theory significantly underpredicts the experimental data, which indicates the need of further improvements in the corresponding nuclear wave functions. In this study, emphasis is placed on the contributions of two-body axial currents that are carefully analyzed using two-body transition densities. This allow us to study the spatial distribution and short-range behavior of two-body dynamics. In particular, the transition densities when scaled to peak at 1.0 exhibit universal short-range behavior across the considered nuclei, while they differ in the long-range tails.

Keywords: nuclear interactions, nuclear currents, chiral effective field theory, ab-initio calculations, weak transitions

\section{INTRODUCTION}

Nuclei are used for high-precision tests of the Standard Model and for studies of physics beyond the Standard Model. Without a thorough understanding of nuclei, we will not be able to meaningfully interpret the growing body of experimental data nor will be able to disentangle new physics signals from underlying nuclear effects. Current and next generation experimental programs are poised to address open questions within fundamental symmetries and neutrino physics to understand the origin of nonzero neutrino masses, the observed matter, and anti-matter unbalance, and the nature of dark matter. These experimental endeavors often rely on accurate calculations of electroweak structure and reactions in nuclei.

For example, nuclear physics plays a pivotal role in searches for neutrinoless double beta $(0 \nu \beta \beta)$ decays, which are the subject of an intense experimental research program [1-14]. In these decays, two neutrons inside the nucleus decay into two protons via the exchange of a neutrino, emitting two electrons. The rates of these decays depend not only on unknown fundamental neutrino parameters but also on nuclear properties. Extracting the neutrino parameters from experiments requires theoretical evaluation of nuclear matrix elements for neutrinoless double beta decay. This 
decay, if observed, would have tremendous theoretical implications and could give insight into our understanding of the observed matter-antimatter asymmetry in the universe. Calculations for nuclei of experimental interest $(A \geq 48)$ are based on computational methods that inevitably adopt approximations to solve the nuclear many-body problem-e.g., model space truncations and/or omission of many-body effects. As a consequence, estimates of $0 v \beta \beta$ matrix elements may vary by a factor of two when computed using different computational models (see [15] and references therein). It is then crucial to understand neutrino-nucleus interactions with great accuracy as well as the role and relevance of many-body dynamics, such as many-nucleon correlations and currents.

Furthermore, intense experimental research activity is currently focused on long-base neutrino oscillation experiments (such as MiniBooNE, T2K, MicroBooNE, Minerva, and the upcoming DUNE; [16-20]) aimed at profiling neutrinos, whose masses, among other properties, are still not known. Neutrinos signal their presence by interacting with nuclei which are the active material in the detectors. Additionally, in this case, meaningful interpretations of the data require an accurate understanding of the way neutrinos interact with nuclei.

The study of light nuclei, for which the nuclear manybody problem can be solved exactly or within controlled approximations by fully retaining many-nucleon correlations and electroweak currents, offers the possibility of quantifying the contribution from many-body effects and consequently of assessing the robustness of a given approximation. In this work, we report on a recent study of electroweak matrix elements in $A \leq 10$ nuclei entering single beta decays and electron captures (or inverse beta decays). Rates of single beta decay-a process in which a proton (neutron) inside the nucleus decays into a neutron (proton) with the emission of a positron (electron) and an electron (anti)neutrino-are, in most cases, experimentally well-known. This provides us with stringent means to validate our theoretical description of nuclear systems and to assess the role of many-body dynamics. In particular, we work within the nuclear microscopic approach in which nuclei are described in terms of non-relativistic nucleons interacting with each other via two- and three-body nuclear potentials and with external probes, such as neutrinos, electrons, and photons, via one- and two-body current operators. We use Quantum Monte Carlo (QMC) computational methods [21-23] to solve the many-body nuclear problem with a nuclear Hamiltonian consisting of highquality two- and three-body potentials obtained from a chiral effective field theory ( $\chi$ EFT) that retains nucleons, pions, and $\Delta$-isobars as explicit degrees of freedom [24-28]. We base the calculation of the transition matrix elements on one- and twobody axial currents [29] and provide results for one- and twobody weak transition densities. The latter will turn out to be particularly important to understand the role of short-range many-body dynamics.

$A b$ initio studies in light nuclei allow us to carefully test many-body correlations and electroweak currents and serve as benchmark to approximated many-body methods currently employed to access heavier nuclear systems [30,31]. A study along these lines has been carried out recently in [32].
This study represents a first step into the validation of our theoretical model. In fact, beta decay processes occur at zero momentum transfer while the energy transfer involved is of the order of a few MeVs. Neutrinos exchanged in $0 v \beta \beta$ processes carry a value of momentum transfer of the order of few hundreds of $\mathrm{MeV} / \mathrm{c}$ [15], while the energy transfer in neutrino oscillation experiments covers a large phase space reaching the $\mathrm{GeV}$ scale. It is then essential to validate the theoretical model in a wide range of energy and momentum transfer to have a complete and unified description of neutrinonucleus interactions. For example, calculations of total and partial muon-capture rates and comparisons with the known experimental data will probe our model at intermediate values of momentum transfer and will be the subject of our future work. At higher energies, neutrino-nucleus cross sections calculations [33, 34] are the main input to interpret the data from long and short baseline neutrino-oscillation experiments, which use nuclei as active material in the detectors. Specifically, current challenges concern the implementation of microscopic models of nuclear dynamics-that fully capture correlation effects-in neutrino event generators [35] used to simulate the neutrino interaction physics.

The paper is structured as follows: In section 2, we briefly summarize the theoretical and computational methods adopted in the present work and refer the interested reader to [36] for further details. In sections 3 and 4, we present our results and conclusions.

\section{THEORY}

\subsection{Quantum Monte Carlo Methods}

Quantum Monte Carlo methods have been most recently described in several review articles [21-23]. Here, we briefly sketch the employed calculational scheme and refer the reader to [36] for details. We use both Variational Monte Carlo (VMC) and Green's Function Monte Carlo (GFMC) methods to calculate transitions matrix elements. We base our study on the following many-body Hamiltonian:

$$
H=\sum_{i} K_{i}+\sum_{i<j} v_{i j}+\sum_{i<j<k} V_{i j k}
$$

where $K_{i}$ is the non-relativistic kinetic energy operator and $v_{i j}$ and $V_{i j k}$ are the NV2 and NV3 Norfolk local chiral interactions developed in [24-28]. Together, we denote these interactions as $\mathrm{NV} 2+3$.

For a nuclear state with given angular momentum and parity $J^{\pi}$, isospin $T$, and isospin projection $T_{Z}$, the VMC method takes as a starting point a trial wave function $\Psi_{V}\left(J^{\pi}, T, T_{z}\right)$, constructed as follows

$$
\left|\Psi_{V}\right\rangle=\mathcal{S} \prod_{i<j}^{A}\left[1+U_{i j}+\sum_{k \neq i, j}^{A} \tilde{U}_{i j k}^{T N I}\right]\left|\Psi_{J}\right\rangle .
$$

The Jastrow wave function $\Psi_{J}$ is fully antisymmetric and has the $\left(J^{\pi} ; T, T_{z}\right)$ quantum numbers of the state of interest, and $U_{i j}$ and 
$\tilde{U}_{i j k}^{T N I}$ are two- and three-body correlation operators that reflect the influence of the two- and three-body forces, respectively [3740]. The state $\left|\Psi_{V}\right\rangle$ has embedded variational parameters that one adjusts to minimize the expectation value

$$
E_{V}=\frac{\left\langle\Psi_{V}|H| \Psi_{V}\right\rangle}{\left\langle\Psi_{V} \mid \Psi_{V}\right\rangle} \geq E_{0}
$$

evaluated with Metropolis Monte Carlo integration [41].

The VMC wave function $\left|\Psi_{V}\right\rangle$ is further improved using the GFMC method. The variational state is propagated in imaginary time with the operator $\exp \left[-\left(H-E_{0}\right) \tau\right]$. This is done in small steps in imaginary time, $\Delta \tau$, and produces the following:

$$
\Psi(\tau)=e^{-\left(H-E_{0}\right) \tau} \Psi_{V}=\left[e^{-\left(H-E_{0}\right) \Delta \tau}\right]^{n} \Psi_{V}
$$

One can see that, for $\tau \rightarrow \infty$, the variational state becomes the desired state $\Psi_{0}$. The evaluation of the off-diagonal expectation value of a given operator $O$ is calculated using the following approximation

$$
\begin{gathered}
\frac{\left\langle\Psi^{f}(\tau)|O| \Psi^{i}(\tau)\right\rangle}{\sqrt{\left\langle\Psi^{f}(\tau) \mid \Psi^{f}(\tau)\right\rangle} \sqrt{\left\langle\Psi^{i}(\tau) \mid \Psi^{i}(\tau)\right\rangle}} \\
\approx\langle O(\tau)\rangle_{M_{i}}+\langle O(\tau)\rangle_{M_{f}}-\langle O\rangle_{V},
\end{gathered}
$$

where $\langle O\rangle_{\mathrm{V}}$ is the variational expectation value and $\langle O(\tau)\rangle_{M}$ is the mixed estimate defined as the following:

$$
\langle O(\tau)\rangle_{M_{f}}=\frac{\left\langle\Psi^{f}(\tau)|O| \Psi_{V}^{i}\right\rangle}{\left\langle\Psi^{f}(\tau) \mid \Psi_{V}^{f}\right\rangle} \sqrt{\frac{\left\langle\Psi_{V}^{f} \mid \Psi_{V}^{f}\right\rangle}{\left\langle\Psi_{V}^{i} \mid \Psi_{V}^{i}\right\rangle}}
$$

and $\langle O(\tau)\rangle_{M_{i}}$ is defined similarly (see [42] for more details).

\subsection{Norfolk Interaction Models}

The calculations of weak transitions presented in this work employ the high-quality local NV2+3 interactions developed in [24-27]. The two-nucleon potentials, NV2s, include a strong interaction component derived from a $\chi$ EFT that involves nucleons, pions, and $\Delta$-isobars as explicit degrees of freedom and an electromagnetic interaction component, including up to terms quadratic in the fine structure constant $\alpha$. The component induced by the strong interaction is separated into long- and short-range parts, labeled $v_{i j}^{L}$ and $v_{i j}^{S}$, respectively. The $v_{i j}^{L}$ part is mediated via one-pion-exchange (OPE) and two-pion-exchange (TPE) terms up to next-to-next-to-leading order (N2LO) in the chiral expansion. Its strength is determined by the nucleon axial coupling $g_{A}$ and the nucleon-to- $\Delta$ axial coupling $h_{A}$, the pion decay amplitude $F_{\pi}$, and LECs $c_{1}, c_{2}, c_{3}, c_{4}$, and $b_{3}+b_{8}$ constrained by fits to $\pi N$ scattering-data [43]. Values of these LECs are provided in Table 1 of [36].

The pion-range operators are strongly singular at short-range in configuration space and are regularized by a radial function that is characterized by a cutoff $R_{L}$ as reported in [24-27]. The $v_{i j}^{S}$ part, however, is described by contact terms up to next-to-nextto-next-to-leading order (N3LO), characterized by 26 unknown
LECs. These interactions have been recently constrained to a large set of $N N$-scattering data, as assembled by the Granada group [44-46], including the deuteron ground-state energy and two-neutron scattering length. For the contact terms, we use a Gaussian representation of the three-dimensional delta function, with $R_{S}$ being the short-range regulator.

In this work, we focus on one class of NV2 interaction, namely the NV2-Ia. This class fits about 2,700 NN scattering data in the range of $0-125 \mathrm{MeV}$ of laboratory energies with a $\chi^{2} /$ datum $\lesssim 1.1[24,25]$. The NV2-Ia uses the combination of short- and long-range regulators $\left(R_{S}, R_{L}\right)=(0.8,1.2) \mathrm{fm}$.

The NV2 models alone are not enough to provide sufficient attraction in GFMC calculations of the binding energies of light nuclei [25]. For this reason, a consistent three-body interaction up to N2LO in the chiral expansion has been developed [47] to go with the two-body potential. This interaction consists of a longrange part mediated by two-pion exchange and a short-range part parameterized in terms of two contact interactions $[48,49]$. The two $3 N$ LECs, namely $c_{D}$ and $c_{E}$, have been obtained either by fitting exclusively strong-interaction observables [47, 50-52] or by relying on a combination of strong- and weak-interaction ones $[27,53,54]$. This last approach is made possible by a relation established in $\chi$ EFT [55] between $c_{D}$ and the LECs entering the contact axial current at N3LO [53, 54], [Schiavilla, private communication].

In [47], the values for $c_{D}$ and $c_{E}$ were obtained by reproducing both the experimental trinucleon ground-state energies and nd doublet scattering length for each of the NV2 models considered. On the other hand, in [27], these LECs were constrained by fitting, in addition to the trinucleon energies, the empirical value of the Gamow-Teller matrix element in tritium $\beta$-decay. The resulting Hamiltonian is denoted as NV2+3-Ia (or Ia for short) in the first case, and as NV2+3-Ia* (or Ia* for short) in the second.

As shown in Table 1, these two different procedures for fixing $c_{D}$ and $c_{E}$ produced rather different values for these LECs, particularly for $c_{E}$ which was found to be relatively large and negative in the unstarred models but quite small, and not consistently negative, in the starred models. This in turn impacts predictions for the spectra of light nuclei [36] and the equation of state of neutron matter, since a negative $c_{E}$ leads to repulsion in light nuclei but attraction in neutron matter [56].

The starred and unstarred NV2+3 Norfolk interactions have been implemented in both the VMC and GFMC codes and used to perform calculations of the energy levels [28, 47], charge radii, and longitudinal elastic form factors [23] of $A=4-12$ nuclei that are found to be in very satisfactory agreement with the experimental data. Furthermore, two of the NV2 $+3 *$ models have been also used to perform VMC calculations of the Fermi, Gamow-Teller, and tensor densities for ${ }^{6} \mathrm{He} \rightarrow{ }^{6} \mathrm{Be}$ and ${ }^{12} \mathrm{Be} \longrightarrow$ ${ }^{12} \mathrm{C}$ transitions [57], relevant for studies of $0 v \beta \beta$.

The NV2 models have recently been used in benchmark calculations of the energy per particle of pure neutron matter as a function of the baryon density using three independent many-body methods: Brueckner-Bethe-Goldstone (BBG), Fermi hypernetted chain/single-operator chain (FHNC/SOC), and AFDMC [58]. The inclusion of three-body forces is essential for a realistic description of neutron matter. These types of calculation 
TABLE 1 $\mid C_{D}$ and $C_{E}$ values of the contact terms in the three-nucleon interactions obtained from fits to (i) the nd scattering length and the trinucleon binding energies [27, 47]; and (ii) the central value of the ${ }^{3} \mathrm{H}$ GT matrix element and the trinucleon binding energies (starred values).

\begin{tabular}{ccc}
\hline & la & \multicolumn{1}{c}{$\mathbf{l a}^{\mathbf{*}}$} \\
\hline$C_{D}$ & 3.666 & -0.635 \\
$C_{E}$ & -1.638 & -0.090 \\
\hline
\end{tabular}

See text and [36] for details.

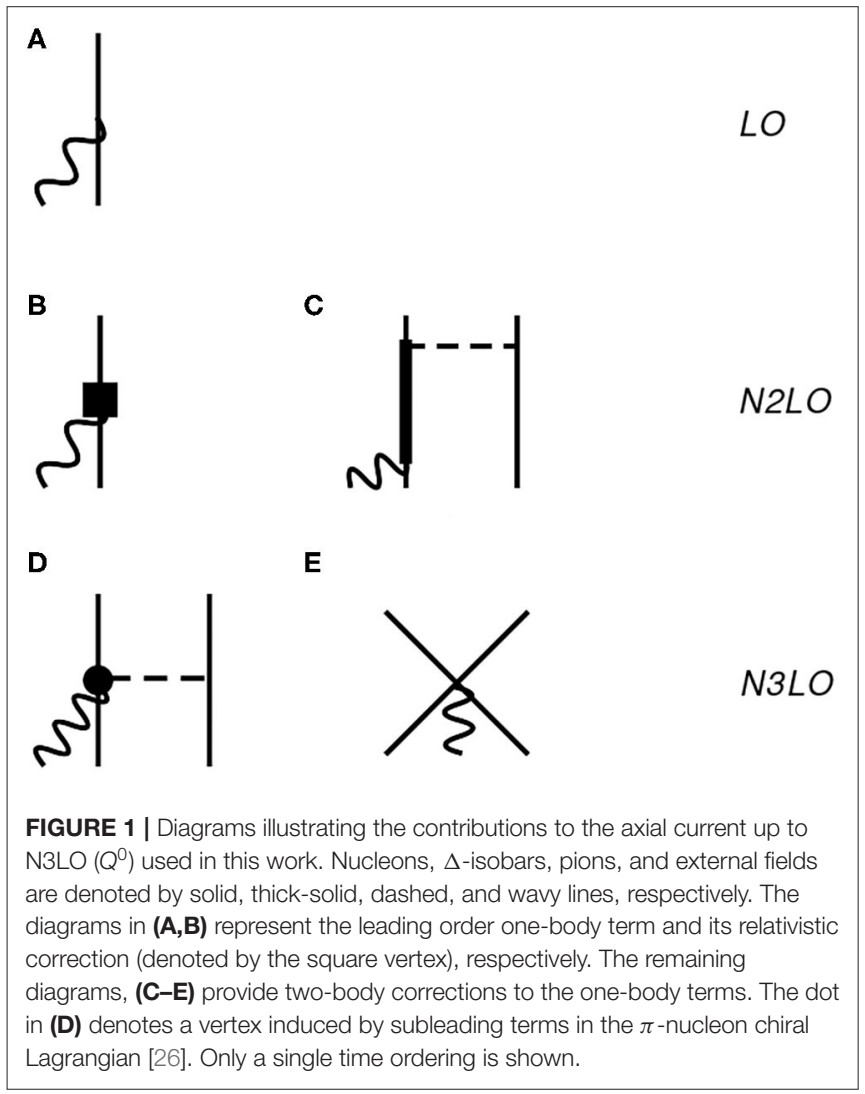

are particularly relevant for the quantitative assessment of the systematic error of the different many-body approaches and how they depend upon the nuclear interaction of choice.

Preliminary AFDMC calculations of the equation of state of pure neutron matter carried out with the unstarred NV2+3 Norfolk interactions [Piarulli et al., private communication] are not compatible with the existence of two solar masses neutron stars, in conflict with recent observations $[59,60]$. However, the smaller values of $c_{E}$ in the $3 \mathrm{~N}$ force of the starred NV2+3 potentials might mitigate, if not resolve this problem, while predicting light-nuclei spectra $<4 \%$ away from the experimental data [36]. Studies along these lines are under way.

\subsection{Axial Currents in $\chi$ EFT}

Many-body currents are crucial for providing a quantitatively successful description of many nuclear electroweak observables [61], such as nuclear electromagnetic form factors [62-65], low-energy electroweak transitions [6673], and electroweak scattering [33]. They have also been used in studies of double beta decay matrix elements [32, 57, 74, 75]. The study of the electroweak matrix elements carried out in this work employs one- and two-body axial currents derived within the same $\chi$ EFT used for the NV2+3 interactions [27]. We use two-body axial currents at tree-level constructed in $[26,27,29]$. Here, we briefly describe the contributions shown in Figure 1 and refer the reader to $[27,36]$ for the explicit expressions of the current operators and for the tables reporting the values of the parameters adopted in the present work. We note that $\chi \mathrm{EFT}$ electroweak operators have been also derived in [76-79].

In Figure 1A, the LO term, which scales as $Q^{-3}$ in the power counting ( $Q$ denotes generically a low-momentum scale), is given by the standard Gamow-Teller one-body operator

$$
\mathbf{j}_{5, a}^{\mathrm{LO}}(\mathbf{q})=-\frac{g_{A}}{2} \tau_{i, a} \boldsymbol{\sigma}_{i} \mathrm{e}^{i \mathbf{q} \cdot \mathbf{r}_{i}},
$$

where $g_{A}$ is the nucleon axial coupling constant $\left(g_{A}=1.2723\right.$ [80]) $\boldsymbol{\sigma}_{i}$ and $\boldsymbol{\tau}_{i}$ are the spin and isospin Pauli matrices of nucleon $i, \mathbf{q}$ is the external field momentum, $\mathbf{r}_{i}$ is the spacial coordinate of nucleon $i$, and $a=x, y, z$.

We account for an additional one body-operator shown in Figure 1B. This contribution enters at N2LO (or $Q^{-1}$ ) in the chiral expansion and represents a relativistic correction to the single-nucleon operator at $\mathrm{LO}$. At N2LO there is the appearance of the leading two-body contribution illustrated in Figure 1C by the tree-level diagram involving the excitation of a $\Delta$-isobar. In the tables and figures below, we label these two contributions as N2LO-RC and N2LO- $\Delta$, respectively. Following the same notation introduced in [27], we write the cumulative N2LO contribution as

$$
\mathbf{j}_{5, a}^{\mathrm{N} 2 \mathrm{LO}}(\mathbf{q})=\mathbf{j}_{5, a}^{\mathrm{N} 2 \mathrm{LO}}(\mathbf{q} ; \mathrm{RC})+\mathbf{j}_{5, a}^{\mathrm{N} 2 \mathrm{LO}}(\mathbf{q} ; \Delta) .
$$

Finally, the N3LO contributions (scaling as $Q^{0}$ ) involve a term of one-pion range illustrated in Figure 1D and a contact term shown in Figure 1E, which together give the following N3LO correction

$$
\mathbf{j}_{5, a}^{\mathrm{N} 3 \mathrm{LO}}(\mathbf{q})=\mathbf{j}_{5, a}^{\mathrm{N} 3 \mathrm{LO}}(\mathbf{q} ; \mathrm{OPE})+\mathbf{j}_{5, a}^{\mathrm{N} 3 \mathrm{LO}}(\mathbf{q} ; \mathrm{CT})
$$

These terms are denoted with N3LO-OPE and N3LOCT, respectively, and their expression are reported in Equations (2.7)-(2.10) of [27]. As discussed at length in [36], the N3LO-CT contact current involves the LEC $c_{D}$, which also enters the three-nucleon force. The values for the LEC $c_{D}$ used in this work are reported in Table $\mathbf{1}$ and are changed consistently in the axial current depending on the nuclear interaction used to construct the wave functions. In this work, we especially focus on the nuclear interactions NV2+3-Ia and NV2+3-Ia*. 
TABLE 2 | Gamow-Teller RMEs in A = 6, 7, 8, and 10 nuclei obtained with chiral axial currents [27] and VMC wave functions corresponding to the NV2+3-la/la* Hamiltonian models $[24,25,27,47]$.

\begin{tabular}{|c|c|c|c|c|c|c|c|c|c|}
\hline Transition & Model & LO & N2LO-RC & N2LO- $\Delta$ & N3LO-OPE & N3LO-CT & (Total-LO) & Total & Expt. \\
\hline${ }^{6} \mathrm{He}\left(0^{+} ; 1\right) \rightarrow{ }^{6} \mathrm{Li}\left(1^{+} ; 0\right)$ & la & 2.200 & -0.016 & 0.037 & 0.039 & -0.005 & 0.056 & 2.256 & $2.1609(40)$ \\
\hline$[42] \rightarrow[42]$ & $\mathrm{la}^{*}$ & 2.192 & -0.015 & 0.036 & 0.038 & -0.054 & 0.005 & 2.197 & \\
\hline${ }^{7} \mathrm{Be}\left(\frac{3}{2}^{-} ; \frac{1}{2}\right) \rightarrow{ }^{7} \mathrm{Li}\left(\frac{3}{2}^{-} ; \frac{1}{2}\right)$ & la & 2.317 & -0.024 & 0.099 & 0.083 & -0.010 & 0.148 & 2.465 & $2.3556(47)$ \\
\hline$[43] \rightarrow[43]$ & $\mathrm{la}^{*}$ & 2.327 & -0.024 & 0.098 & 0.082 & -0.121 & 0.036 & 2.362 & \\
\hline${ }^{7} \mathrm{Be}\left(\frac{3}{2}^{-} ; \frac{3}{2}\right) \rightarrow{ }^{7} \mathrm{Li}\left(\frac{1}{2}^{-} ; \frac{1}{2}\right)$ & la & 2.157 & 0.000 & 0.066 & 0.063 & -0.009 & 0.121 & 2.278 & $2.1116(57)$ \\
\hline$[43] \rightarrow[43]$ & $\mathrm{la}^{*}$ & 2.158 & 0.000 & 0.065 & 0.063 & -0.103 & 0.026 & 2.184 & \\
\hline${ }^{8} \mathrm{Li}\left(2^{+} ; 1\right) \rightarrow{ }^{8} \mathrm{Be}\left(2^{+} ; 0\right)$ & la & 0.147 & 0.000 & 0.032 & 0.011 & -0.001 & 0.041 & 0.188 & 0.284 [84] \\
\hline$[431] \rightarrow[44]$ & $\mathrm{la}^{*}$ & 0.141 & 0.000 & 0.031 & 0.010 & -0.017 & 0.025 & 0.166 & 0.190 [85] \\
\hline${ }^{8} \mathrm{~B}\left(2^{+} ; 1\right) \rightarrow{ }^{8} \mathrm{Be}\left(2^{+} ; 0\right)$ & la & 0.146 & 0.000 & 0.032 & 0.011 & -0.001 & 0.042 & 0.188 & $0.269(20)$ \\
\hline$[431] \rightarrow[44]$ & $\mathrm{la}^{*}$ & 0.148 & 0.000 & 0.032 & 0.010 & -0.016 & 0.026 & 0.174 & \\
\hline${ }^{8} \mathrm{He}\left(0^{+} ; 2\right) \rightarrow{ }^{8} \mathrm{Li}\left(1^{+} ; 1\right)$ & la & 0.386 & -0.004 & 0.034 & 0.009 & -0.001 & 0.038 & 0.424 & $0.512(6)$ \\
\hline$[422] \rightarrow[431]$ & $\mathrm{la}^{*}$ & 0.362 & -0.004 & 0.035 & 0.009 & -0.010 & 0.029 & 0.391 & \\
\hline${ }^{10} \mathrm{C}\left(\mathrm{O}^{+} ; 1\right) \rightarrow{ }^{10} \mathrm{~B}\left(1^{+} ; 0\right)$ & la & 1.940 & -0.024 & 0.026 & 0.042 & -0.006 & 0.039 & 1.9879 & $1.8331(34)$ \\
\hline$[442] \rightarrow[442]$ & $\mathrm{la}^{*}$ & 2.051 & -0.012 & 0.020 & 0.039 & -0.065 & -0.017 & 2.033 & \\
\hline
\end{tabular}

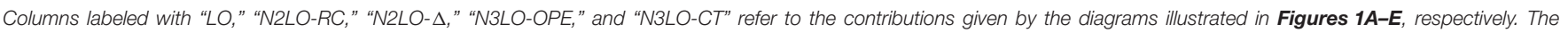

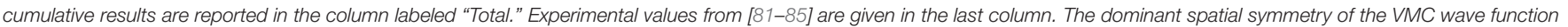

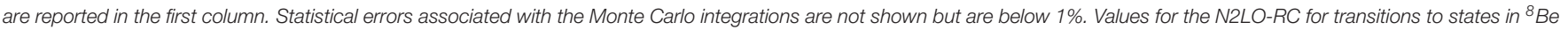
and to the state ${ }^{7} \mathrm{Li}\left(\frac{3}{2}^{-} ; \frac{1}{2}\right)$ are 0.000 within the statistical uncertainty of the integration.

\section{RESULTS}

\subsection{VMC Reduced Matrix Elements}

In this section, we present the results of calculations of GT reduced matrix elements (RMEs), defined as the following:

$$
\mathrm{RME}=\frac{\sqrt{2 J_{f}+1}}{g_{A}} \frac{\left\langle J_{f} M\left|j_{5, \pm}^{z}\right| J_{i} M\right\rangle}{\left\langle J_{i} M, 10 \mid J_{f} M\right\rangle}
$$

where $j_{5, \pm}^{z}$ is the $z$-component in the limit $\mathbf{q} \rightarrow 0$ of the chargeraising/lowering current $\mathbf{j}_{5, \pm}=\mathbf{j}_{5, x} \pm i \mathbf{j}_{5, y}$, and $\left\langle J_{i} M, 10 \mid J_{f} M\right\rangle$ is a Clebsch-Gordan coefficient. Table 2 summarizes the results of the VMC calculation of GT RMEs. These calculations were evaluated with variational wave functions generated using the NV2+3-Ia and NV2+3-Ia* nuclear Hamiltonians. The table breaks the calculations down order-by-order: the LO contribution from the one-body axial current in Figure 1A, the N2LO contributions coming from a one-body relativistic correction to the LO term (Figure 1B) and a two-body contribution involving the excitation of a nucleon into a $\Delta$ by pion exchange (Figure 1C), and the contributions at N3LO from the one pion exchange (Figure 1D) and the contact term (Figure 1E). The sum of the two body contributions (Total-LO) and the total RME are given in addition to the breakdown of each contribution. The dominant spatial symmetries [86] of the wave functions in each calculation are listed below the transition in Table 2. Experimental values from [81-85] are listed in the last column of Table 2.

From these results, we see that in the $A=3,6,7$, and 10 cases, the LO contribution is $\approx 97 \%$ of the total RME in VMC calculations. The other $\simeq 3 \%$ are made up of beyond leading order contributions. While beyond leading order contributions only make up a small percentage of the total RME for the $A=$
$3,6,7$, and 10 cases, they are a much larger contribution for $A=$ 8 , making up $\sim 20-30 \%$ of the total RME. This is attributed to a difference in the dominant spatial symmetries between the initial and final states of the $A=8 \mathrm{VMC}$ wave functions, resulting in a smaller overlap between the initial and final wave functions and a consequent suppression of the GT RME at leading order. This indicates that the wave functions are lacking correlations and that an improvement of the theoretical prediction will require further developments of the wave functions, such as the inclusion of more correlations and development of better constrained small components. As similar behavior is also found in the calculations of [87]. The total two-body contribution is typically an enhancement of the total RME, except in the $A=10$ case for model NV2+3-Ia*, where the matrix element is reduced. The short-range behavior of the two-body corrections is studied in detail in section 3.2 where we analyze the two-body transition densities.

For ${ }^{8} \mathrm{Li} \rightarrow{ }^{8}$ Be beta decay, there are two different $\log (f t)$ values in the literature that provide very different values for the GT matrix element. In Table 2, we present the RMEs for this decay using the $f t$ values from $[84,85]$, obtained with the following formula [83]:

$$
\operatorname{RME}(\operatorname{EXPT})=\frac{1}{g_{A}} \sqrt{2 J_{i}+1} \sqrt{\frac{6139 \pm 7}{f t}},
$$

where $J_{i}$ is the angular momentum of the initial state. Note that the Fermi transition strength is negligible in deriving Equation (11). This formula uses the value $g_{A}=1.2723$. Additionally, we note that in $[82,88]$, a value of 6,147 is used in place of 6,139 . Even with this uncertainty in the experimental 
value of the RME, our predictions for the $A=8$ systems significantly underestimate the data.

\subsection{One- and Two-Body Transition Densities}

To investigate the behavior of individual contributions to the RMEs, one- and two-body transition densities are calculated. One can define the one-body transition density as a function of the distance of nucleon $i$ from the center of mass:

$$
\operatorname{RME}(1 \mathrm{~b})=\int \mathrm{d} r_{i} 4 \pi r_{i}^{2} \rho^{1 \mathrm{~b}}\left(r_{i}\right) .
$$

In Figures 2, 3, the one-body transition density is found to be consistent between the two cases. Indeed, this is what we expect based on Table 2. The LO contribution is consistent between the two interactions and is not sensitive to how the LECs of the three-body interaction are fit. Looking at the sub-leading order contributions, we find that, with the exception of the N2LO-RC term in the $A=10$ transitions, the only term that is model dependent is the N3LO contact term. To better understand this difference, in a fashion similar to what is done in Equation (12) for the one-body case, a two-body transition density as a function of inter-particle spacing $r_{i j}$ can be defined:

$$
\operatorname{RME}(2 \mathrm{~b})=\int \mathrm{d} r_{i j} 4 \pi r_{i j}^{2} \rho^{2 \mathrm{~b}}\left(r_{i j}\right)
$$

Two-body transition densities are plotted in Figure 4. For the same transition, the N2LO- $\Delta$ and the N3LO-OPE contributions in models NV2+3-Ia and NV2+3-Ia* are nearly identical. This is to be expected, as the two models use the same cutoffs to regularize the interactions and have the two-body interaction fit to the same data. Where the models differ is in the N3LO-CT contribution. Model NV2+3 $\mathrm{Ia}^{\star}$ has a larger contribution from this term compared to its counterpart. This model-dependence of the contact contribution to the RME makes sense in light of the difference between models NV2+3-Ia and NV2+3-Ia*. The LECs $c_{D}$ and $c_{E}$ entering the three-body contact current were fit with two different procedures. In model NV2+3 Ia, the contribution was constrained using only strong interaction data while in model NV2+3 $\mathrm{Ia}^{*}$, both strong and electroweak data were used to constrain it. This results in the two models having different values for these LECs and thus different strengths in the contact term. While there is evidently a model-dependence, it is worth noting that this is a small contribution to the overall RME for the transitions.

Although there is a model dependence in the N3LO-CT term, it is interesting to ask if the behavior of the current is still similar between the two models. For this purpose, in Figure 5, transition densities for the N3LO-CT current selected nuclei are scaled to peak at 1.0 to see if there is a universal behavior in the interactions. The scaling factors to generate Figure 5 are given in Table 3. While there was a difference seen in the size of the contribution of the N3LO-CT term when comparing the unscaled transition densities, it is seen here that the scaled curve overlaps for not only both models within the same transition but

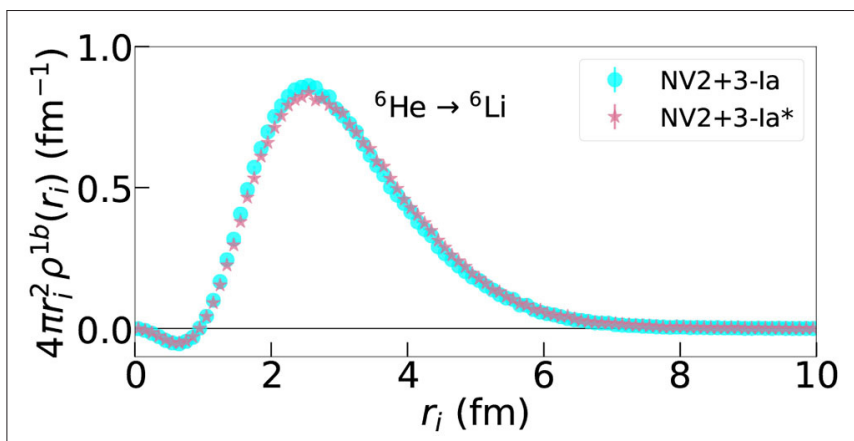

FIGURE 2 | One-body density as defined in Equation (12) for the ${ }^{6} \mathrm{He} \rightarrow{ }^{6} \mathrm{Li}$ GT RME obtained using the NV2+3-la/la* models.

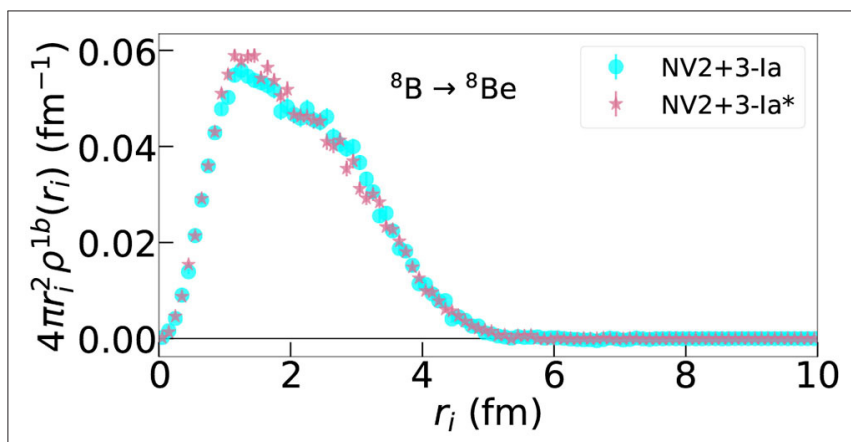

FIGURE 3 | One-body density as defined in Equation (12) for the ${ }^{8} \mathrm{~B} \rightarrow{ }^{8} \mathrm{Be}$ GT RME obtained using the NV2+3-la/la* models.

also for all transitions under study. The change in the LECs $c_{D}$ and $c_{E}$ results in a re-scaling of the N3LO-CT term. For the $A \leq 7$ transitions, the enhancement of the N3LO-CT term relative in $\mathrm{NV} 2+3-\mathrm{Ia}^{*}$ relative to the value given by its counterpart $\mathrm{NV} 2+3-$ Ia is a factor of $\approx 6.4$ for the same transition. For the $A \geq 8$ cases, this enhancement is on average a factor of $\approx 2.2$.

Another important feature of the two-body transition densities is the difference in the long-range behavior of the N2LO- $\Delta$ and N3LO-OPE terms. In [27], Equations (2.9) and (2.10) give the operator structure of these two currents. In the limit of vanishing momentum transfer, these currents have the same operator structure up to a momentum dependent term that has been verified numerically to provide small contributions. The structures of these operators result in cancelations that are sensitive to the LECs entering into each of these currents, impacting the behavior of the two-body transition density for $r_{i j} \gtrsim 2 \mathrm{fm}$. In particular, the N2LO- $\Delta$ density is sensitive to the transition under study. In the case of the $A=10$ transition, the N2LO- $\Delta$ density becomes negative near $\approx 2$ $\mathrm{fm}$. When integrating over the whole two-body contribution for the NV2+3-Ia* model, this results in a non-trivial cancelation leading to the quenching of the RME from the inclusion of sub-leading contributions. 


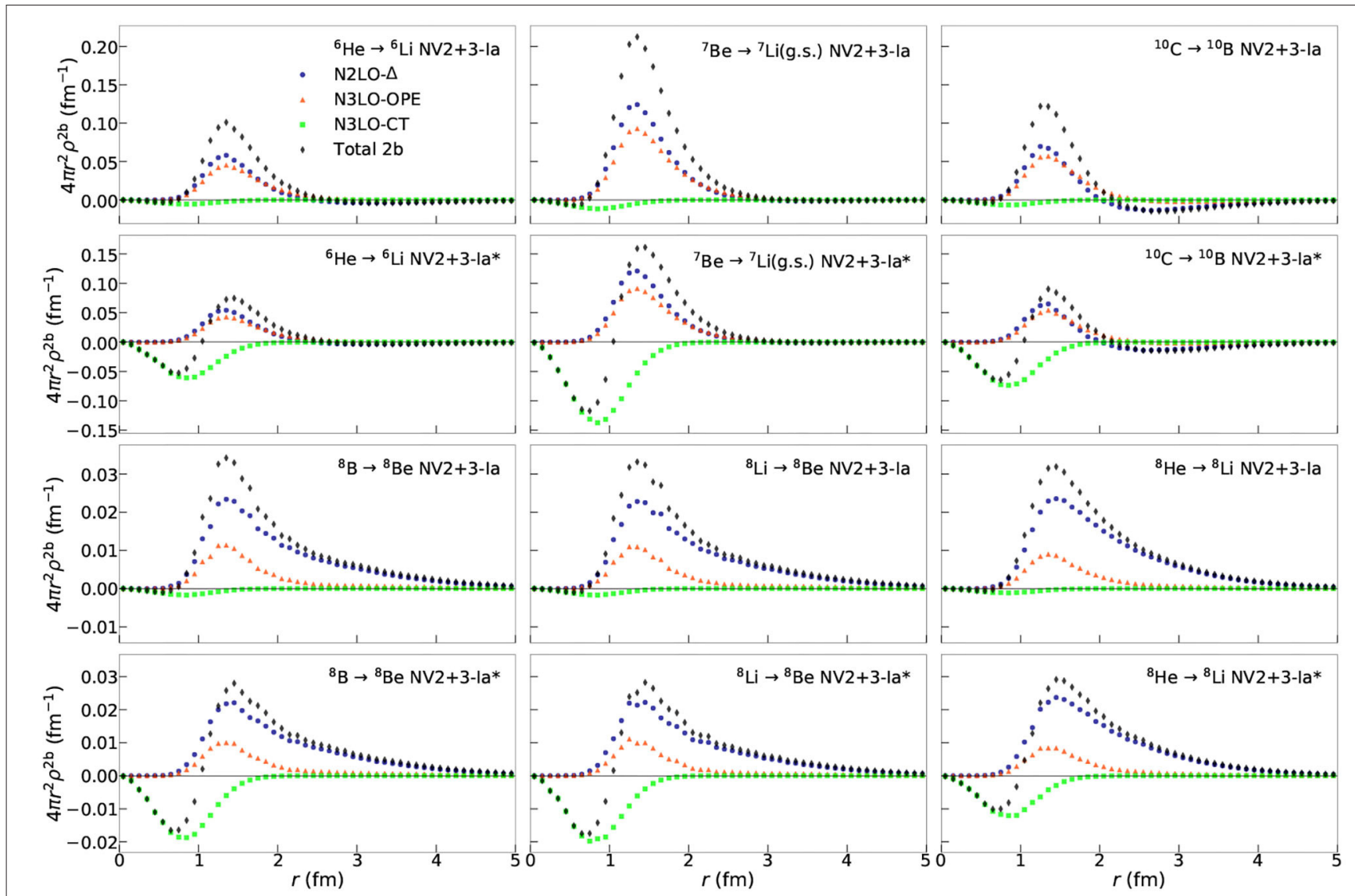

FIGURE 4 | Two-body density as defined in Equation (13) for GT RMEs obtained using the NV2+3-la/la* models for select nuclei.

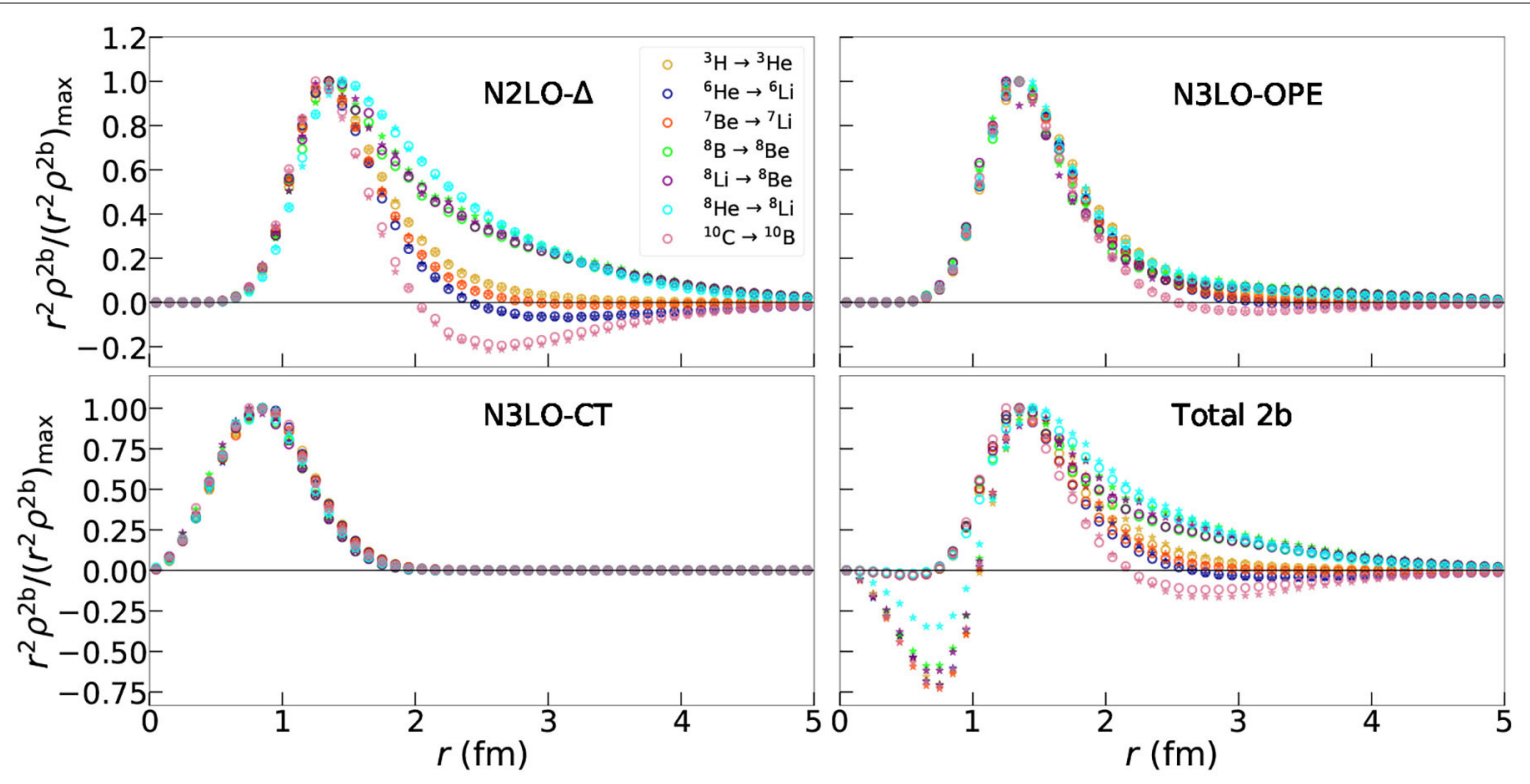

FIGURE 5 | Scaled two-body density as defined in Equation (13) for GT RMEs obtained using the NV2+3-la/la* models for select nuclei. The distributions are scaled to have a peak value of 1.0 using the factors in Table 3. 


\subsection{GFMC Extrapolation}

In addition to VMC calculations of the RME, we also perform a GFMC extrapolation of the RME. The GFMC wave functions generated with the NV2+3-Ia model in this work produce energies that are in statistical agreement with the results of [47]. For all cases presented below, with the exception of two transitions, GFMC propagation are performed between imaginary time steps $\tau=0.2$ and $0.82 \mathrm{MeV}^{-1}$. Typically, an imaginary time evolution of the VMC estimate produces an RME that is reduced by a few percent and stabilizes at $\tau \approx 0.2 \mathrm{MeV}^{-1}$.

TABLE 3 | The scaling factors $\left(r_{i j}^{2} \rho^{2 b}\right)_{\max }$ used to normalize the N2LO- $\Delta$, N3LO-OPE, N3LO-CT, and total two-body transition densities in Figure $\mathbf{5}$.

\begin{tabular}{lccccc}
\hline Transition & Model & N2LO- $\Delta$ & N3LO-OPE & N3LO-CT & Total 2b \\
\hline${ }^{3} \mathrm{H} \rightarrow{ }^{3} \mathrm{He}$ & $\mathrm{la}$ & 0.111 & 0.082 & -0.010 & 0.189 \\
& $\mathrm{la} \mathrm{a}^{*}$ & 0.107 & 0.081 & -0.120 & 0.147 \\
${ }^{6} \mathrm{He} \rightarrow{ }^{6} \mathrm{Li}$ & $\mathrm{la}$ & 0.058 & 0.045 & -0.005 & 0.101 \\
& $\mathrm{la} \mathrm{a}^{*}$ & 0.054 & 0.043 & -0.061 & 0.075 \\
${ }^{7} \mathrm{Be} \rightarrow{ }^{7} \mathrm{Li}$ & $\mathrm{la}$ & 0.124 & 0.093 & -0.012 & 0.212 \\
& $\mathrm{la}{ }^{*}$ & 0.121 & 0.091 & -0.137 & 0.161 \\
${ }^{8} \mathrm{~B} \rightarrow{ }^{8} \mathrm{Be}$ & $\mathrm{la}$ & 0.023 & 0.011 & -0.002 & 0.034 \\
& $\mathrm{la}{ }^{*}$ & 0.022 & 0.010 & -0.019 & 0.028 \\
${ }^{8} \mathrm{Li} \rightarrow{ }^{8} \mathrm{Be}$ & $\mathrm{la}$ & 0.023 & 0.011 & -0.002 & 0.033 \\
& $\mathrm{la}{ }^{*}$ & 0.022 & 0.011 & -0.020 & 0.028 \\
${ }^{8} \mathrm{He} \rightarrow{ }^{8} \mathrm{Li}$ & $\mathrm{la}$ & 0.023 & 0.009 & -0.001 & 0.032 \\
& $\mathrm{la}{ }^{*}$ & 0.037 & 0.008 & -0.012 & 0.029 \\
${ }^{10} \mathrm{C} \rightarrow{ }^{10} \mathrm{~B}$ & $\mathrm{la}$ & 0.070 & 0.057 & -0.006 & 0.122 \\
& $\mathrm{la}{ }^{*}$ & 0.061 & 0.050 & -0.073 & 0.085
\end{tabular}

See text for details.
In the calculations of transitions involving the $\left(J^{\pi} ; T\right)=\left(2^{+} ; 0\right)$ state of ${ }^{8} \mathrm{Be}$ and the ground state of ${ }^{8} \mathrm{~B}$, the extrapolation must be treated differently. In this two states, as $\tau$ increases, the binding energy, magnitude of the quadrupole moment, and point-proton radius all increase monotonically. This is interpreted as the dissolution of ${ }^{8} \mathrm{Be}$ into two alpha particles and ${ }^{8} \mathrm{~B}$ into $p+{ }^{7} \mathrm{Be}$. Datar et al. [71], Pastore et al. [72], and Wiringa et al. [89] have previously addressed this issue for ${ }^{8} \mathrm{Be}$. Similar to those references, we perform the extrapolation by noting that the energy drops rapidly in $\tau$, stabilizing at $\tau \approx 0.1 \mathrm{MeV}^{-1}$. We assume that, at this point, spurious contamination in the wave function has been removed by the GFMC procedure and average in a small interval around $\tau=0.1 \mathrm{MeV}^{-1}$, taken to be $\tau$ from 0.06 to $0.14 \mathrm{MeV}^{-1}$. This introduces and additional $\approx 5 \%$ systematic uncertainty to these calculations in addition to the statistical uncertainties of QMC.

In all transitions, except for the NV2+3-Ia* model for $A=10$, the GFMC extrapolation reduces the VMC RME by a $\lesssim 4 \%$. Table 4 summarizes results of the LO, total sub-leading order (Total-LO), and total RMEs for the transitions under study. In the $A<10$ transitions, the $\mathrm{LO}$ contribution is consistent between the two different models under study. In the $A=10$ case, this model dependence can be understood by the existence of nearby $J^{\pi}=1^{+}$ excited states in ${ }^{10} \mathrm{~B}$. The lower state is predominantly ${ }^{3} \mathrm{~S}_{1}[442]$ state and the upper one ${ }^{3} \mathrm{D}_{1}[442]$ state. These two states are split by only $1 \mathrm{MeV}$. The transition from the predominantly ${ }^{1} \mathrm{~S}_{0}[442]$ ${ }^{10} \mathrm{C}\left(0^{+}\right)$state is large in the $S \rightarrow S$ components but about five times smaller in the $S \rightarrow D$ components. This causes the GT matrix element to be particularly sensitive to the exact mixing of the ${ }^{3} \mathrm{~S}_{1}$ and ${ }^{3} \mathrm{D}_{1}$ components in the two ${ }^{10} \mathrm{~B}\left(1^{+}\right)$states produced by a given Hamiltonian, as was observed for the calculation of GT matrix elements using the AV18+IL7 interaction [73]. In either case, the NV2+3 interactions overpredict the data.

TABLE 4 | Gamow-Teller RMEs in A=6, 7, 8, and 10 nuclei obtained with chiral axial currents [27] and GFMC (VMC) wave functions corresponding to the NV2+3-la/la* Hamiltonian models [24, 25, 27, 47].

\begin{tabular}{|c|c|c|c|c|c|}
\hline Transition & Model & LO & (Total-LO) & Total & Expt. \\
\hline$[42] \rightarrow[42]$ & $\mathrm{la}^{*}$ & 2.107 (2.192) & $0.011(0.005)$ & $2.118(2.197)$ & \\
\hline$[43] \rightarrow[43]$ & $\mathrm{la}^{*}$ & $2.286(2.327)$ & $0.052(0.053)$ & $2.338(2.380)$ & \\
\hline${ }^{7} \mathrm{Be}\left(\frac{3}{2}^{-} ; \frac{1}{2}\right) \rightarrow^{7} \mathrm{Li}\left(\frac{1}{2}^{-} ; \frac{1}{2}\right)$ & la & $2.065(2.157)$ & $1.03(0.121)$ & $2.168(2.278)$ & $2.1116(57)$ \\
\hline$[431] \rightarrow[44]$ & $\mathrm{la}^{*}$ & $0.096(0.148)$ & $0.025(0.026)$ & $0.120(0.174)$ & $0.190[85]$ \\
\hline${ }^{8} \mathrm{~B}\left(2^{+} ; 1\right) \rightarrow{ }^{8} \mathrm{Be}\left(2^{+} ; 0\right)$ & la & $0.091(0.146)$ & $0.035(0.042)$ & $0.125(0.188)$ & $0.269(20)$ \\
\hline$[431] \rightarrow[44]$ & $\mathrm{la}^{*}$ & $0.102(0.148)$ & $0.024(0.026)$ & $0.126(0.174)$ & \\
\hline${ }^{8} \mathrm{He}\left(0^{+} ; 2\right) \rightarrow{ }^{8} \mathrm{Li}\left(1^{+} ; 1\right)$ & la & $0.262(0.386)$ & $0.040(0.038)$ & $0.302(0.424)$ & $0.512(6)$ \\
\hline$[422] \rightarrow[431]$ & $\mathrm{la}^{*}$ & $0.297(0.362)$ & 0.025 (0.029) & $0.322(0.391)$ & \\
\hline
\end{tabular}

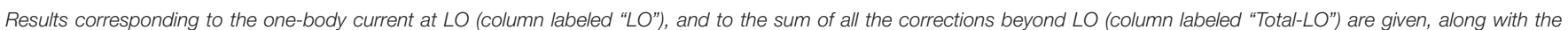

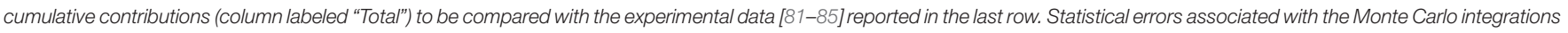
are not shown, but are below $1 \%$. Transitions for the $A=8$ systems are affected by an additional systematic error of $\sim \%$, see text for explanation. 


\section{CONCLUSIONS}

With this work we set the foundations for the development of an accurate and unified understanding of neutrino-nucleus interactions. We are in the process of exploring and validating the QMC approach's description of electroweak processes in a wide range of energy and momentum transfer; in this work, we therefore focused on calculating matrix elements entering beta decay and inverse beta decay in light nuclei. These processes occur at zero momentum transfer and involve energy transferred of the order of a few MeVs.

In our approach, we fully retained two- and three-nucleon correlations induced by the Norfolk potentials, and we described the interaction with the external electroweak probes by means of the associated one- and two-body axial currents at tree-level. This study was focused on the NV2+3-Ia and NV2+3-Ia* models, and was aimed at carefully studying the contributions from two-body axial currents in the two different implementations of the three-nucleon forces. In the unstarred model the LECs $c_{D}$ and $c_{E}$ entering the three-nucleon force were fitted to the trinucleon binding energies and the nd scattering length, while the starred model is constrained by the experimental GT value of the triton decay and the trinucleon binding energies. The axial two-body contact current at N3LO, which involves $c_{D}$, was taken consistently with the three-nucleon force adopted to generate the nuclear wave functions.

In analogy with previous QMC studies of beta decay in light nuclei $[36,73]$, we find that corrections from two-body axial currents are at the $\sim 3 \%$ level in $A=6,7$ and 10 . The $A=8$ systems are instead severely underpredicted by the theory, even after the inclusion of large $(\sim 30-40 \%)$ contributions from twobody axial currents. Studies on the electromagnetic transitions in $A=8$ nuclei were also found to be problematic [70, 72], which indicates the need of further developments of the $A=8$ wave functions.

In this work, we especially focused on the contributions from two-body currents, which, despite the fact that they are in these cases small, can provide us with valuable insights on the composition of these corrections. To this end, we reported studies on two-body transition densities which allow us to understand

\section{REFERENCES}

1. Gando A, Gando Y, Hachiya T, Hayashi A, Hayashida S, Ikeda H, et al. Publisher's note: search for majorana neutrinos near the inverted mass hierarchy region with KamLAND-Zen [Phys. Rev. Lett. 117, 082503 (2016)]. Phys Rev Lett. (2016) 117:109903. doi: 10.1103/PhysRevLett.117. 082503

2. Albert JB, Auty DJ, Barbeau P, Beauchamp E. Search for Majorana neutrinos with the first two years of EXO-200 data. Nature. (2014) 510:229-34. doi: $10.1038 /$ nature 13432

3. Adams C, Alvarez V, Arazi L, Arnquist IJ, Azevedo CDR, Bailey K, et al. Sensitivity of a tonne-scale NEXT detector for neutrinoless double beta decay searches. arXiv [Preprint] arXiv:2005.06467. (2020)

4. Zsigmond AJ. LEGEND: The future of neutrinoless double-beta decay search with germanium detectors. J Phys Conf Ser. (2020) 1468:012111. doi: $10.1088 / 1742-6596 / 1468 / 1 / 012143$ the relevance of the two-body currents as a function of the interparticle distance. As expected, we find that, for a given interaction model, the transition densities exhibit a universal short-range behavior across the considered nuclei, while they differ in the long-range tails. The starred and unstarred results differ in the contact contribution at N3LO. This is rather visible in the panels of Figure $\mathbf{4}$ where the starred model leads to a total transition density (black symbols) which presents one or more nodes. The presence of nodes implies non-trivial cancelations when using the starred models.

\section{DATA AVAILABILITY STATEMENT}

All datasets generated for this study are included in the article/supplementary material.

\section{AUTHOR CONTRIBUTIONS}

All authors listed have made a substantial, direct and intellectual contribution to the work, and approved it for publication.

\section{FUNDING}

This research was supported by the U.S. Department of Energy, Office of Science, Office of Nuclear Physics, under the U.S. Department of Energy funds through the FRIB Theory Alliance award DE-SC0013617 (MP and SP). LA and SP have been supported by the U.S. Department of Energy under contract DE-SC0021027.

\section{ACKNOWLEDGMENTS}

We thank our collaborators J. Carlson, S. Gandolfi, R. Schiavilla, and R. B. Wiringa for their contributions to the studies presented in this work. The many-body calculations were performed on the parallel computers of the Laboratory Computing Resource Center, Argonne National Laboratory, the computers of the Argonne Leadership Computing Facility (ALCF) via the ALCC2019 grant Low energy neutrino-nucleus interactions.

5. Cattadori CM. Gerda: results and perspectives. Nucl Part Phys Proc. (2015) 265-6:38-41. doi: 10.1016/j.nuclphysbps.2015.06.010

6. Alfonso K, Artusa DR, Avignone FT, Azzolini O, Balata M, Banks TI, et al. Search for neutrinoless double-beta decay of ${ }^{1} 30 \mathrm{Te}$ with CUORE-0. Phys Rev Lett. (2015) 115:102502. doi: 10.1103/PhysRevLett.115.102502

7. Caden E. Status of the SNO+ experiment. J Phys Conf Ser. (2020) 1342:012022. doi: 10.1088/1742-6596/1342/1/012022

8. Blot $\mathrm{S}$. Investigating $\beta \beta$ decay with the NEMO-3 and SuperNEMO experiments. J Phys Conf Ser. (2016) 718:062006. doi: 10.1088/1742-6596/718/6/062006

9. Giuliani A. A neutrinoless double-beta-decay search based on $\mathrm{ZnMoO}_{4}$ and $\mathrm{Li}_{2} \mathrm{MoO}_{4}$ scintillating bolometers. J Phys Conf Ser. (2017) 888:012239. doi: 10.1088/1742-6596/888/1/012239

10. Tetsuno K, Ajimura S, Akutagawa K, Batpurev T, Chan WM, Fushimi K, et al Status of 48Ca double beta decay search and its future prospect in CANDLES J Phys Conf Ser. (2020) 1468:012132. doi: 10.1088/1742-6596/1468/1/012132 
11. Park H. The AMoRE: Search for neutrinoless double beta decay in 100Mo. Nucl Part Phys Proc. (2016) 273-5:2630-2. doi: 10.1016/j.nuclphysbps.2015.10.012

12. Ebert J, Fritts M, Gehre D, Gößling C, Göpfert T, Hagner C, et al. The COBRA demonstrator at the LNGS underground laboratory. Nucl Instrum Meth A. (2016) 807:114-20. doi: 10.1016/j.nima.2015.10.079

13. Dokania N, Singh V, Ghosh C, Mathimalar S, Garai A, Pal S, et al. Radiation background studies for $0 v \beta \beta$ decay in ${ }^{1} 24 \mathrm{Sn}$. In: Topical Research Meeting on Prospects in Neutrino Physics. London, UK (2015).

14. Fukuda Y. ZICOS - New project for neutrinoless double beta decay experiment using zirconium complex in liquid scintillator. J Phys. (2016) 718:062019. doi: 10.1088\%2F1742-6596\%2F718\%2F6\%2F062019

15. Engel J, Menndez J. Status and future of nuclear matrix elements for neutrinoless double-beta decay: a review. Rept Prog Phys. (2017) 80:046301. doi: 10.1088/1361-6633/aa5bc5

16. [Dataset] The MicroBooNE Experiment. Available online at: http://wwwmicroboone.fnal.gov

17. [Dataset] The T2K Experiment. Available online at: http://t2k-experiment.org

18. [Dataset] The Deep Underground Neutrino Experiment. Available online at: http://www.dunescience.org

19. [Dataset] The NOvA Experiment. Available online at: http://www-nova.fnal. gov

20. [Dataset] Hyper-Kamiokande. Available online at: http://www.hyperk.org

21. Carlson J, Gandolfi S, Pederiva F, Pieper SC, Schiavilla R, Schmidt KE, et al. Quantum Monte Carlo methods for nuclear physics. Rev Mod Phys. (2015) 87:1067. doi: 10.1103/RevModPhys.87.1067

22. Lynn JE, Tews I, Gandolfi S, Lovato A. Quantum Monte Carlo methods in nuclear physics: recent advances. Ann Rev Nucl Part Sci. (2019) 69:279-305. doi: 10.1146/annurev-nucl-101918-023600

23. Gandolfi S, Lonardoni D, Lovato A, Piarulli M. Atomic nuclei from quantum Monte Carlo calculations with chiral EFT interactions. (2020) Front Phys. 8:117. doi: 10.3389/fphy.2020.00117

24. Piarulli M, Girlanda L, Schiavilla R, Navarro Pérez R, Amaro JE, Ruiz Arriola E. Minimally nonlocal nucleon-nucleon potentials with chiral twopion exchange including $\Delta$ resonances. Phys Rev C. (2015) 91:024003. doi: 10.1103/PhysRevC.91.024003

25. Piarulli M, Girlanda L, Schiavilla R, Kievsky A, Lovato A, Marcucci LE, et al. Local chiral potentials with $\Delta$-intermediate states and the structure of light nuclei. Phys Rev C. (2016) 94:054007. doi: 10.1103/PhysRevC.94.054007

26. Baroni A, Girlanda L, Kievsky A, Marcucci LE, Schiavilla R, Viviani M. Tritium $\beta$-decay in chiral effective field theory. Phys Rev C. (2016) 94:024003. doi: 10.1103/PhysRevC.94.024003

27. Baroni A, Schiavilla R, Marcucci LE, Girlanda L, Kievsky A, Lovato A, et al. Local chiral interactions, the tritium Gamow-Teller matrix element, and the three-nucleon contact term. Phys Rev C. (2018) 98:044003. doi: 10.1103/PhysRevC.98.044003

28. Piarulli M, Tews I. Local nucleon-nucleon and three-nucleon interactions within chiral effective field theory. Front Phys. (2020) 7:245. doi: $10.3389 /$ fphy. 2019.00245

29. Baroni A, Girlanda L, Pastore S, Schiavilla R, Viviani M. Nuclear axial currents in chiral effective field theory. Phys Rev C. (2016) 93:015501. doi: 10.1103/PhysRevC.93.049902

30. Coraggio L, Gargano A, Itaco N, Mancino R, Nowacki F. The calculation of the neutrinoless double-beta decay matrix element within the realistic shell model. Phys Rev C. (2020) 101:044315. doi: 10.1103/PhysRevC.101.044315

31. Coraggio L, Itaco N, Mancino R. Short-range correlations for neutrinoless double-beta decay and low-momentum NN potentials. In: 27th International Nuclear Physics Conference. Glasgow, UK (2019).

32. Wang XB, Hayes AC, Carlson J, Dong GX, Mereghetti E, Pastore S, et al. Comparison between variational Monte Carlo and shell model calculations of neutrinoless double beta decay matrix elements in light nuclei. Phys Lett B. (2019) 798:134974. doi: 10.1016/j.physletb.2019.134974

33. Pastore S, Carlson J, Gandolfi S, Schiavilla R, Wiringa RB. Quasielastic lepton scattering and back-to-back nucleons in the short-time approximation. Phys Rev C. (2019) 101:044612. doi: 10.1103/PhysRevC.101.044612

34. Lovato A, Carlson J, Gandolfi S, Rocco N, Schiavilla R. Ab initio study of $\left(v_{\ell}, \ell^{-}\right)$and $\left(\bar{v}_{\ell}, \ell^{+}\right)$inclusive scattering in ${ }^{1} 2 \mathrm{C}$ : confronting the MiniBooNE and T2K CCQE data. Phys Rev X. (2020) 3:031068. doi: 10.1103/PhysRevX.10.031068

35. Alvarez-Ruso L, Athar MS, Barbaro MB, Cherdack D, Christy ME, Coloma P, et al. NuSTEC1 1Neutrino scattering theory experiment collaboration http://nustec.fnal.gov. White Paper: Status and challenges of neutrino-nucleus scattering. Prog Part Nucl Phys. (2018) 100:1-68. doi: 10.1016/j.ppnp.2018.01.006

36. King GB, Andreoli L, Pastore S, Piarulli M, Schiavilla R, Wiringa RB, et al. Chiral effective field theory calculations of weak transitions in light nuclei. Phys Rev C. (2020) 102:025501. doi: 10.1103/PhysRevC.102.025501

37. Wiringa RB. Variational calculations of few-body nuclei. Phys Rev C. (1991) 43:1585-98. doi: 10.1103/PhysRevC.43.1585

38. Pudliner BS, Pandharipande VR, Carlson J, Pieper SC, Wiringa RB. Quantum Monte Carlo calculations of nuclei with A $<=7$. Phys Rev C. (1997) 56:1720-50. doi: 10.1103/PhysRevC.56.1720

39. Nollett KM, Wiringa RB, Schiavilla R. A Six body calculation of the alpha deuteron radiative capture cross-section. Phys Rev C. (2001) 63:024003. doi: 10.1103/PhysRevC.63.024003

40. Nollett KM. Radiative alpha capture cross-sections from realistic nucleonnucleon interactions and variational Monte Carlo wave functions. Phys Rev C. (2001) 63:054002. doi: 10.1103/PhysRevC.63.054002

41. Metropolis N, Rosenbluth AW, Rosenbluth MN, Teller AH, Teller E. Equation of state calculations by fast computing machines. J Chem Phys. (1953) 21:1087-92. doi: 10.1063/1.1699114

42. Pervin M, Pieper SC, Wiringa RB. Quantum Monte Carlo calculations of electroweak transition matrix elements in $\mathrm{A}=6,7$ nuclei. Phys Rev C. (2007) 76:064319. doi: 10.1103/PhysRevC.76.064319

43. Krebs H, Epelbaum E, Meissner UG. Nuclear forces with Delta-excitations up to next-to-next-to-leading order. I. Peripheral nucleon-nucleon waves. Eur Phys J A. (2007) 32:127-37. doi: 10.1140/epja/i2007-10372-y

44. Navarro Pérez R, Amaro JE, Ruiz Arriola E. Coarse-grained potential analysis of neutron-proton and proton-proton scattering below the pion production threshold. Phys Rev C. (2013) 88:064002. doi: 10.1103/PhysRevC.88.064002

45. Navarro Pérez R, Amaro JE, Ruiz Arriola E. Coarse grained NN potential with chiral two pion exchange. Phys Rev C. (2014) 89:024004. doi: 10.1103/PhysRevC.89.024004

46. Navarro Perez R, Amaro JE, Ruiz Arriola E. Statistical error analysis for phenomenological nucleon-nucleon potentials. Phys Rev C. (2014) 89:064006. doi: 10.1103/PhysRevC.89.064006

47. Piarulli M, Baroni A, Girlanda L, Kievsky A, Lovato A, Lusk E, et al. Light-nuclei spectra from chiral dynamics. Phys Rev Lett. (2018) 120:052503. doi: 10.1103/PhysRevLett.120.052503

48. van Kolck U. Few nucleon forces from chiral Lagrangians. Phys Rev C. (1994) 49:2932-41. doi: 10.1103/PhysRevC.49.2932

49. Epelbaum E, Nogga A, Gloeckle W, Kamada H, Meissner UG, Witala H. Three nucleon forces from chiral effective field theory. Phys Rev C. (2002) 66:064001. doi: 10.1103/PhysRevC.66.064001

50. Lynn JE, Tews I, Carlson J, Gandolfi S, Gezerlis A, Schmidt KE, et al. Chiral three-nucleon interactions in light nuclei, neutron- $\alpha$ scattering, and neutron matter. Phys Rev Lett. (2016) 116:062501. doi: 10.1103/PhysRevLett.116.062501

51. Tews I, Gandolfi S, Gezerlis A, Schwenk A. Quantum Monte Carlo calculations of neutron matter with chiral three-body forces. Phys Rev C. (2016) 93:024305. doi: 10.1103/PhysRevC.93.024305

52. Lynn JE, Tews I, Carlson J, Gandolfi S, Gezerlis A, Schmidt KE, et al. Quantum Monte Carlo calculations of light nuclei with local chiral two- and three-nucleon interactions. Phys Rev C. (2017) 96:054007. doi: 10.1103/PhysRevC.96.054007

53. Gazit D, Quaglioni S, Navratil P. Three-nucleon low-energy constants from the consistency of interactions and currents in chiral effective field theory. Phys Rev Lett. (2009) 103:102502. doi: 10.1103/PhysRevLett.103.102502

54. Marcucci LE, Kievsky A, Rosati S, Schiavilla R, Viviani M. Chiral effective field theory predictions for muon capture on deuteron and ${ }^{3}$ He. Phys Rev Lett. (2012) 108:052502. doi: 10.1103/PhysRevLett.108.052502

55. Gardestig A, Phillips DR. How low-energy weak reactions can constrain threenucleon forces and the neutron-neutron scattering length. Phys Rev Lett. (2006) 96:232301. doi: 10.1103/PhysRevLett.96.232301 
56. Lovato A, Benhar O, Fantoni S, Schmidt KE. Comparative study of three-nucleon potentials in nuclear matter. Phys Rev C. (2012) 85:024003. doi: 10.1103/PhysRevC.85.024003

57. Cirigliano V, Dekens W, De Vries J, Graesser ML, Mereghetti E, Pastore S, et al. A renormalized approach to neutrinoless double-beta decay. Phys Rev C. (2019) 100:055504. doi: 10.1103/PhysRevC.100.055504

58. Piarulli M, Bombaci I, Logoteta D, Lovato A, Wiringa RB. Benchmark calculations of pure neutron matter with realistic nucleon-nucleon interactions. Phys Rev C. (2019) 101:045801. doi: 10.1103/PhysRevC.101.045801

59. Demorest P, Pennucci T, Ransom S, Roberts M, Hessels J. Shapiro delay measurement of a two solar mass neutron star. Nature. (2010) 467:1081-3. doi: 10.1038/nature09466

60. Antoniadis J, Freire PCC, Wex N, Tauris TM, Lynch RS, van Kerkwijk MH, et al. A massive pulsar in a compact relativistic binary. Science. (2013) 340:6131. doi: $10.1126 /$ science. 1233232

61. Bacca S, Pastore S. Electromagnetic reactions on light nuclei. J Phys G. (2014) 41:123002. doi: 10.1088/0954-3899/41/12/123002

62. Piarulli M, Girlanda L, Marcucci LE, Pastore S, Schiavilla R, Viviani M. Electromagnetic structure of $\mathrm{A}=2$ and 3 nuclei in chiral effective field theory. Phys Rev C. (2013) 87:014006. doi: 10.1103/PhysRevC.87.014006

63. Schiavilla R, Baroni A, Pastore S, Piarulli M, Girlanda L, Kievsky A, et al. Local chiral interactions and magnetic structure of few-nucleon systems. Phys Rev $C$. (2019) 99:034005. doi: 10.1103/PhysRevC.99.034005

64. Nevo Dinur N, Hernandez OJ, Bacca S, Barnea N, Ji C, Pastore S, et al. Zemach moments and radii of ${ }^{2}, 3 \mathrm{H}$ and ${ }^{3}, 4 \mathrm{He}$. Phys Rev C. (2019) 99:034004. doi: 10.1103/PhysRevC.99.034004

65. Marcucci LE, Gross F, Pena MT, Piarulli M, Schiavilla R, Sick I, et al. Electromagnetic structure of few-nucleon ground states. J Phys G. (2016) 43:023002. doi: 10.1088/0954-3899/43/2/023002

66. Pastore S, Schiavilla R, Goity JL. Electromagnetic two-body currents of one- and two-pion range. Phys Rev C. (2008) 78:064002. doi: 10.1103/PhysRevC.78.064002

67. Pastore S, Girlanda L, Schiavilla R, Viviani M, Wiringa RB. Electromagnetic currents and magnetic moments in (chi)EFT. Phys Rev C. (2009) 80:034004. doi: 10.1103/PhysRevC.80.034004

68. Girlanda L, Kievsky A, Marcucci LE, Pastore S, Schiavilla R, Viviani M. Thermal neutron captures on $d$ and ${ }^{3} \mathrm{He}$. Phys Rev Lett. (2010) 105:232502. doi: 10.1103/PhysRevLett.105.232502

69. Pastore S, Girlanda L, Schiavilla R, Viviani M. The two-nucleon electromagnetic charge operator in chiral effective field theory $(\chi \mathrm{EFT})$ up to one loop. Phys Rev C. (2011) 84:024001. doi: 10.1103/PhysRevC.84.024001

70. Pastore S, Pieper SC, Schiavilla R, Wiringa RB. Quantum Monte Carlo calculations of electromagnetic moments and transitions in $\mathrm{A} \leq 9$ nuclei with meson-exchange currents derived from chiral effective field theory. Phys Rev C. (2013) 87:035503. doi: 10.1103/PhysRevC.87.035503

71. Datar VM, Chakrabarty DR, Suresh Kumar, Nanal V, Pastore S, Wiringa RB, et al. Electromagnetic transition from the $4+$ to $2+$ resonance in Be8 measured via the radiative capture in He4+He4. Phys Rev Lett. (2013) 111:062502. doi: 10.1103/PhysRevLett.111.062502

72. Pastore S, Wiringa RB, Pieper SC, Schiavilla R. Quantum Monte Carlo calculations of electromagnetic transitions in ${ }^{8} \mathrm{Be}$ with meson-exchange currents derived from chiral effective field theory. Phys Rev C. (2014) 90:024321. doi: 10.1103/PhysRevC.90.024321

73. Pastore S, Baroni A, Carlson J, Gandolfi S, Pieper SC, Schiavilla R, et al. Quantum Monte Carlo calculations of weak transitions in $A=6-10$ nuclei. Phys Rev C. (2018) 97:022501. doi: 10.1103/PhysRevC.97.022501

74. Pastore S, Carlson J, Cirigliano V, Dekens W, Mereghetti E, Wiringa RB. Neutrinoless double- $\beta$ decay matrix elements in light nuclei. Phys Rev $C$. (2018) 97:014606. doi: 10.1103/PhysRevC.97.014606
75. Cirigliano V, Dekens W, De Vries J, Graesser ML, Mereghetti E, Pastore S, et al. New leading contribution to neutrinoless double- $\beta$ decay. Phys Rev Lett. (2018) 120:202001. doi: 10.1103/PhysRevLett.120. 202001

76. Krebs H, Epelbaum E, Meißner UG. Box diagram contribution to the axial two-nucleon current. Phys Rev C. (2020) 101:055502. doi: 10.1103/PhysRevC.101.055502

77. Park TS, Min DP, Rho M. Chiral dynamics and heavy fermion formalism in nuclei. 1. Exchange axial currents. Phys Rept. (1993) 233:341-95. doi: 10.1016/0370-1573(93)90099-Y

78. Kolling S, Epelbaum E, Krebs H, Meissner UG. Two-pion exchange electromagnetic current in chiral effective field theory using the method of unitary transformation. Phys Rev C. (2009) 80:045502. doi: 10.1103/PhysRevC.80.045502

79. Kolling S, Epelbaum E, Krebs H, Meissner UG. Two-nucleon electromagnetic current in chiral effective field theory: one-pion exchange and short-range contributions. Phys Rev C. (2011) 84:054008. doi: 10.1103/PhysRevC.84.054008

80. Tanabashi M, Hagiwara K, Hikasa K, Nakamura K, Sumino Y, Takahashi F, et al. Review of particle physics. Phys Rev D. (2018) 98:030001. doi: 10.1103/PhysRevD.98.030001

81. Knecht A, Hong R, Zumwalt DW, Delbridge BG, Garcia A. Precision measurement of the He-6 half-life and the weak axial current in nuclei. Phys Rev C. (2012) 86:035506. doi: 10.1103/PhysRevC.86.035506

82. Suzuki T, Fujimoto R, Otsuka T. Gamow-Teller transitions and magnetic properties of nuclei and shell evolution. Phys Rev C. (2003) 67:044302. doi: 10.1103/PhysRevC.67.044302

83. Chou WT, Warburton EK, Brown BA. Gamow-teller beta-decay rates for A $<=18$ nuclei. Phys Rev C. (1993) 47:163-77. doi: 10.1103/PhysRevC. 47.163

84. Warburton EK. R-matrix analysis of the $\beta^{\mp}$-delayed alpha spectra from the decay of ${ }^{8} \mathrm{Li}$ and ${ }^{8} \mathrm{~B}$. Phys Rev C. (1986) 33:303-13. doi: 10.1103/PhysRevC.33.303

85. Pritychenko B, BK E, Kellett MA, Singh B, Totans J. The Nuclear Science References (NSR) database and Web Retrieval System. Nucl Instrum Methods Phys Res Sect A. (2011) 640:213-8. doi: 10.1016/j.nima.2011.03.018

86. Wiringa RB. Pair counting, pion-exchange forces, and the structure of light nuclei. Phys Rev C. (2006) 73:034317. doi: 10.1103/PhysRevC.73.034317

87. Gysbers P, Hagen G, Holt JD, Jansen GR, Morris TD, Navratil P, et al. Discrepancy between experimental and theoretical $\beta$-decay rates resolved from first principles. Nat Phys. (2019) 15:428-31. doi: 10.1038/s41567-019-0450-7

88. Hardy JC, Towner IS. Superallowed $0^{+} \rightarrow 0^{+}$nuclear decays: 2014 critical survey, with precise results for $V_{u} d$ and CKM unitarity. Phys Rev C. (2015) 91:025501. doi: 10.1103/PhysRevC.91.025501

89. Wiringa RB, Pieper SC, Carlson J, Pandharipande VR. Quantum Monte Carlo calculations of A $=8$ nuclei. Phys Rev C. (2000) 62:014001. doi: 10.1103/PhysRevC.62.014001

Conflict of Interest: The authors declare that the research was conducted in the absence of any commercial or financial relationships that could be construed as a potential conflict of interest.

Copyright $\odot 2020$ King, Andreoli, Pastore and Piarulli. This is an open-access article distributed under the terms of the Creative Commons Attribution License (CC BY). The use, distribution or reproduction in other forums is permitted, provided the original author(s) and the copyright owner(s) are credited and that the original publication in this journal is cited, in accordance with accepted academic practice. No use, distribution or reproduction is permitted which does not comply with these terms. 